\title{
Model Identification and Attitude Control for a Micromechanical Flying Insect Including Thorax and Sensor Models
}

\author{
Xinyan Deng, Luca Schenato and Shankar S. Sastry \\ Dept. of EECS, University of California, Berkeley, CA 94720 \\ $\{$ xinyan, lusche, sastry\}@eecs.berkeley.edu
}

\begin{abstract}
This paper describes recent developments on the model identification and attitude control system for a Micromechanical Flying Insect (MFI). We include recently developed dynamical models for the thorax actuators and various sensor models. Wing kinematic parameterization scheme was designed to generate feasible wing motions to decouple the body torques under the constraints of the thorax model. A nominal state-space LTI model in hover was identified through linear estimation and a LQR controller was designed to achieve stable hovering and steering maneuvers. Simulation results show satisfactory performance comparable to that of the real insects.
\end{abstract}

\section{INTRODUCTION}

Most research work on Micro aerial vehicles (MAVs) today are based on fixed and rotary wings [1]. However, flapping wing flight observed in insects demonstrates superior performance and maneuverability. The aim of the UC Berkeley Micromechanical Flying Insect (MFI) project is to use biomimetic principles to develop a MAV that can achieve exceptional flight performance through the use of flapping wings [2]

Biomimetic sensor models, including halteres, ocelli, and magnetic compass to measure angles and angular rates in body frame are recently developed, together with a linear thorax actuator model. Due to the constraints on the input amplitude of the thorax model, low level wing motion design and open loop control problem is reconsidered. The original wing motion parameterization method in our previous work [3] is no longer feasible since discontinuities at the end of consecutive wingbeats cause the thorax input torques to jump and saturate. In this work, we describe a new wing kinematic parameterization method, which keeps the thorax inputs smooth and bounded, while still decouples roll, pitch, yaw body torques.

Based on this parameterization, we adopt our previous identification and control scheme [3] to find an equivalent discrete-time linear time-invariant (LTI) model to approximate the main dynamics of the MFI near hover and design a sensor feedback LQR controller which stabilizes the MFI and provides setpoint tracking.

\section{ATTITUdE DYNAMICS}

The attitude dynamics of a flying insect is described as

$$
\begin{aligned}
\dot{R} & =R \hat{\omega}^{b} \\
\dot{\omega}^{b} & =J^{-1}\left(\tau^{b}-\omega^{b} \times J \omega^{b}\right) \\
\hat{\omega}^{b} & =\left[\begin{array}{ccc}
0 & \omega_{z}^{b} & -\omega_{y}^{b} \\
-\omega_{z}^{b} & 0 & \omega_{x}^{b} \\
\omega_{y}^{b} & -\omega_{x}^{b} & 0
\end{array}\right]
\end{aligned}
$$

where $\omega^{b}=\left[\omega_{x}^{b} \omega_{y}^{b} \omega_{z}^{b}\right]^{T}$ is the body frame angular velocity vector, $\tau^{b} \in \mathbb{R}^{3}$ is the body frame aerodynamic torque vector, $J \in \mathbb{R}^{3 \times 3}$ is the insect body moment of inertia. To simplify the notation, we drop the superscript $b$ from equations, implicitly assuming that all quantities are measured relative to the body frame $B$, attached to the center of mass. $R \in S O(3)=\left\{R \in \mathbb{R}^{3 \times 3}: R^{T} R=I\right.$, det $R=$ $+1\}$ is the rotation matrix representing the orientation of the insect body frame $B$ relative to the fixed frame $A$. In particular, we parametrize the rotation matrix $R$ by $\operatorname{roll}(\eta)$, $\operatorname{pitch}(\theta), y a w(\psi)$ Euler angles.

\section{SENSORS}

Three sensors, namely, the ocelli, the magnetic compass and the halteres, are developed to estimate the roll, pitch, yaw angles and angular velocities in body frame. In this paper we report only the major results and details are presented in [4]. These sensors are currently being built and preliminary results are very promising [5].

\section{A. Ocelli}

The ocelli can be used to estimate roll and pitch angles through light detection. It is a sensory system present in many flying insects which comprise of three wide angle photoreceptors placed on the head of the insect. They are oriented in such a way to collect light from different regions of the sky. By comparing the light intensity measured by different photoreceptors it helps to achieve attitude stabilization in insect flight [6].

We developed a biomimetic ocelli-like sensor system composed of four ideal photoreceptors, denoted $P_{1}, P_{2}, P_{3}, P_{4}$, fixed with respect to the body frame $B$. They are oriented symmetrically to have the same latitude and their axes intersect the sky sphere forming an imaginary pyramid, whose vertex is placed at the center of the insect head. Every photoreceptor collects light from a conic 


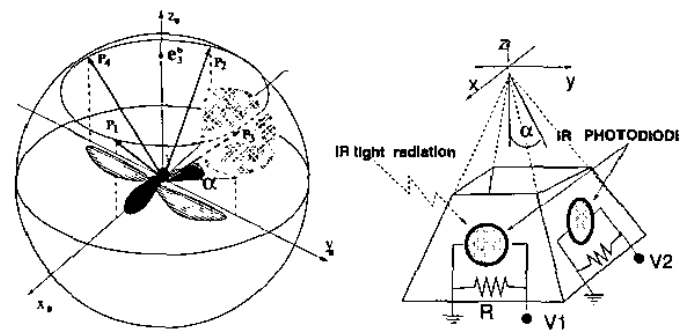

Fig. 1. (a) Graphical rendering of ocelli present on flying insects. Four photoreceptors, $P_{1}, P_{2}, P_{3}, P_{4}$, collect light from different regions of the sky. The shadowed area represents the region for photoreceptor $P_{3}$. (b) Schematic of ocelli biomimetic sensor.

region $A_{i}$ around its ideal orientation $P_{i}$ as shown in Figure 1 .

The measurements from the photoreceptors are simply subtracted pairwise and form the outputs:

$$
y_{1}^{o}=I\left(P_{1}\right)-I\left(P_{2}\right), \quad y_{2}^{o}=I\left(P_{3}\right)-I\left(P_{4}\right)
$$

where $I\left(P_{i}\right)$ is the output from the $i$-th photodiode. Without loss of generality we assume that the light source is placed at position $X=(0,0,1)$ relative to the fixed frame. If the output of a photodiode is a monotonic decreasing function of its latitude relative to the light source, it was showed that the following proposition holds true [4]:

Proposition 1: If the photoreceptor output is a monotonic decreasing function of its latitude $\theta_{i}$ relative to the light source, then the signals $y_{1}^{o}$ and $y_{2}^{o}$ defined in Equations (2) always satisfy the following conditions:

$$
\begin{aligned}
& k_{\min } r_{32} \leq y_{1}^{o} \leq k_{\max } r_{32} \\
& k_{\min } r_{31} \leq y_{2}^{o} \leq k_{\max } r_{31} \\
r_{33} \rightarrow 1 \Longrightarrow & y_{1}^{o} \rightarrow k_{o} r_{32} ; y_{2}^{o} \rightarrow k_{o} r_{31}
\end{aligned}
$$

where $0<k_{\min }<k_{o}<k_{\max }$ are constant, and $r_{i j}$ is the $i-j$ entry of the rotation matrix $R$.

Therefore, it is evident that the outputs from the ocelli can be used as an estimate of the position of the ocelli reference frame relative to the light source, since for small deviations from the equilibrium, i.e. $R \approx I_{3 \times 3}, r_{31}$ and $r_{32}$ correspond to the roll and pitch angles, respectively. Detailed results are given in [4]. A prototype for the ocelli system, which is currently being developed [5] seems to confirm the mathematical model results.

\section{B. MEMS Magnetic Compass}

The MEMS magnetic compass is used to estimate MFI heading (yaw angle) based on the terrestrial geomagnetic field. It is a "U-shaped" suspended structure as shown in Figure 2. As electric current flows across this structure, it interacts with the terrestrial geomagnetic field, and the Lorentz forces is generatesd by $\mathbf{F}_{l}=L \mathbf{i} \times \mathbf{B}$, where $\mathbf{F}_{l}$ is the force at the tip of the cantilever, $L$ is the length

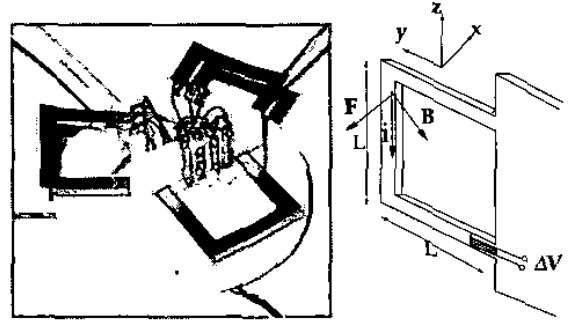

Fig. 2. (a) Photo of a MEMS magnetic sensor prototype (b) Schematic of a magnetic compass.

of one side of the cantilever, $\mathbf{i}$ is the current, and $\mathbf{B}$ is the terrestrial electromagnetic field. The deflection of the cantilever is sensed at the base by a strain gage, and is proportional to the force perpendicular to the cantilever,i.e. $F_{c}=\mathbf{F}_{l} \cdot \mathbf{n}$, where $\mathbf{n}$ is the sensing direction. Therefore, the output from the strain gage can be used to estimate the heading of the MFI when the magnetic sensor is attached to the MFI body. Without loss of generality, assume that the earth magnetic field is oriented along the $x$-axis of the fixed frame, i.e. $\mathbf{B}=[00 \mathrm{~B}]^{T}$ relative to the fixed frame. If we orient the compass such that the current $\mathbf{i}=\left[\begin{array}{lll}0 & 0 & -i\end{array}\right]^{T}$ and the sensing direction $\mathbf{n}=\left[\begin{array}{lll}1 & 0 & 0\end{array}\right]^{T}$ relative to the body frame (see Figure 2), then the signal measured by the strain gage is given by:

$$
\begin{aligned}
y^{c} & =F_{c}=L\left(\left[\begin{array}{lll}
0 & 0 & -i
\end{array}\right] \times\left(R^{T}\left[\begin{array}{lll}
0 & 0 & B
\end{array}\right]^{T}\right)\right)\left[\begin{array}{lll}
1 & 0 & 0
\end{array}\right]^{T} \\
& =L i B r_{12}=k_{c} r_{12}
\end{aligned}
$$

where we use the fact that the coordinates of the earth magnetic field relative to the body frame is given by $v^{b}=$ $R^{T}\left[\begin{array}{lll}0 & 0 & B\end{array}\right]^{T}$. For small deviations from the equilibrium, i.e. $R \approx I_{3 \times 3}$, and $r_{12}$ correspond to the yaw angle, thus providing an estimate for the heading.

\section{Halteres}

Halteres are used to measure angular velocities in the body frame. Biomechanical studies revealed that insects use halteres to measure body rotations via gyroscopic forces [7]. The halteres of a fly resemble small balls at the end of thin sticks. During flight the two halteres beat up and down in non-coplanar planes through an angle of nearly $180^{\circ}$ anti-phase to the wings at the wingbeat frequency. This non-coplanarity of the two halteres is essential for a fly to detect rotations about all three turning axes [8]

During insect flight, forces acting on the halteres include inertial, angular acceleration, centrifugal, Coriolis, and gravitational forces. However, particular characteristics (frequency, modulation, and phase) of the Coriolis signals on the left and right halteres motivated a demodulation scheme that can decipher roll, pitch, and yaw angular velocities [5]. It was shown [4] that the halteres outputs are almost equivalent to the following smoothed version 


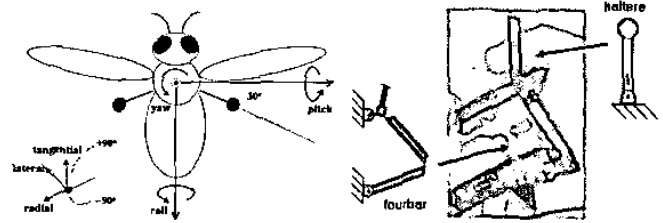

Fig. 3. (a) Schematic of enlarged halteres of a fly. (b) Photo of the completed haltere.

of the insect angular velocities:

$$
\begin{aligned}
& y_{h}^{h}(t)=a \int_{t-T}^{t} \omega_{x}(\tau) \mathrm{d} \tau=k_{o 1} \bar{\omega}_{x}(t) \\
& y_{2}^{h}(t)=b \int_{t-T}^{t} \omega_{y}(\tau) \mathrm{d} \tau=k_{o 2} \bar{\omega}_{y}(t) \\
& y_{3}^{h}(t)=c \int_{t-T}^{t} \omega_{z}(\tau) \mathrm{d} \tau=k_{o 3} \bar{\omega}_{z}(t)
\end{aligned}
$$

where $T$ is the period of oscillation, $a, b, c, k_{o 1}, k_{o 2}, k_{o 3}$ are constants and $\bar{\omega}_{i}$ is the mean angular velocity of the insect over a period of oscillation of the halteres.

\section{Linear THORAX MODEL}

Each wing is controlled by a thorax, a complex trapezoidal structure actuated by two piezoelectric actuators at its base, as shown in Figure 4. A complete nonlinear model for the thorax, developed in [9], can be written as

$$
M\left[\begin{array}{c}
\ddot{\theta}_{2} \\
\ddot{\alpha}
\end{array}\right]+B\left[\begin{array}{c}
\dot{\theta}_{2} \\
\dot{\alpha}
\end{array}\right]+K\left[\begin{array}{c}
\theta_{2} \\
\alpha
\end{array}\right]+\left[\begin{array}{c}
0 \\
f(\dot{\alpha})
\end{array}\right]=T\left[\begin{array}{l}
u_{1} \\
u_{2}
\end{array}\right]_{(8)}
$$

where $f(\dot{\alpha})=\frac{1}{2} m_{\omega, 2}^{\prime}(\dot{\alpha})^{2}, \theta_{2}$ is the leading edge flapping angle from the four bar mechanism, $\alpha=\theta_{1}-\theta_{2}$ is the phase difference between the four bar output angles, $u_{1}$ and $u_{2}$ are the control input torques to the actuators, $M$ and $B$ are the inertia and damping matrices. Parameters in $K$ and $T$ matrices includes some slowly time varying terms, and the control inputs $\left(u_{1}, u_{2}\right)$ are limited to $10 \mu \mathrm{Nm}$ by physical constraints.

The relation between the state variables in Equation (8) and the wing motion variables (stroke angle $\phi$, rotation angle $\varphi$ ) can be approximated as $\phi=\theta_{2}$ and $\varphi=2 \alpha$. Based on Equation (8), with a change of variables, neglecting the nonlinear components, we can derive the linear actuator model as

$$
M_{0}\left[\begin{array}{c}
\ddot{\phi} \\
\ddot{\phi}
\end{array}\right]+B_{0}\left[\begin{array}{c}
\dot{\phi} \\
\dot{\varphi}
\end{array}\right]+K_{0}\left[\begin{array}{l}
\phi \\
\varphi
\end{array}\right]=T_{0}\left[\begin{array}{l}
u_{1} \\
u_{2}
\end{array}\right]
$$

where $M_{0}, B_{0}, K_{0}$, and $T_{0}$ are constant matrices.

Equation (9) is a stable linear MIMO system, and its steady state solution at a particular frequency can be calculated through complex matrix operations. To generate sufficient lift to sustain the insect, the stroke and rotation angles must follow a trajectory which mimic insect wing flapping motions, such as $\phi=\frac{\pi}{3} \cos (w t)$ and $\varphi=\frac{\pi}{4} \sin (w t)$, where $w=2 \pi f$ and $f=150 \mathrm{~Hz}$ is the wingbeat frequency. The desired steady state input torques to the actuators that generate the above wing trajectories can be calculated from

$$
\left[\begin{array}{l}
u_{1} \\
u_{2}
\end{array}\right]=\left[\begin{array}{l}
G_{11}(j w) G_{12}(j w) \\
G_{21}(j w) G_{22}(j w)
\end{array}\right]_{w=150 * 2 \pi}^{-1}\left[\begin{array}{c}
\frac{\pi}{3} e^{j \pi / 2} \\
\frac{\pi}{4}
\end{array}\right]
$$

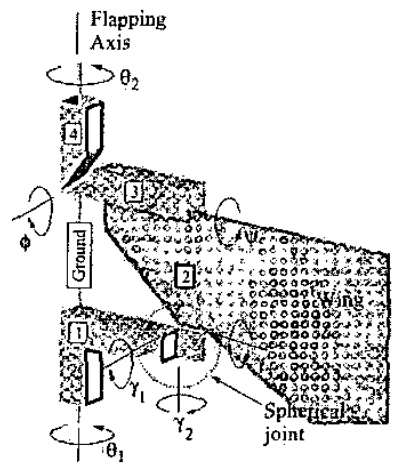

Fig. 4. Wing-Thorax structure. Courtesy of [9]

where $G(j w)$ is the frequency domain system transfer function matrix, the resulted steady state inputs are $u_{1}=$ $5.64 \sin (w t-2.67), u_{2}=6.48 \sin (w t-2.47)$. These inputs drive the wings to their steady state trajectory within $2-3$ wingbeats, when the wings are started from rest.

\section{WING MOTION PARAMETERIZATION}

In order to decouple roll, pitch, yaw body toques, we need to manipulate wing motions through proper kinematic parameterization schemes. Meanwhile, smooth and bounded input torques to the thorax is desired. One feasible parameterization was found by adding an additional term, $g(t)$, in the stroke angle profile to change the wing flapping amplitude, and in the rotation angle profile to change the wing rotation timing at the end of the first half-stroke.

$$
\begin{aligned}
g(t) & =\frac{3}{10} \sin \left(\frac{1}{2} w t\right)-\frac{1}{10} \sin \left(\frac{3}{2} w t\right) \\
\phi_{l}(t) & =\frac{\pi}{3}\left(\cos (w t)+\frac{1}{2} \gamma g(t)\right) \\
\phi_{r}(t) & =\frac{\pi}{3}\left(\cos (w t)-\frac{1}{2} \gamma g(t)\right) \\
\varphi_{l}(t) & =\frac{\pi}{4}\left(\sin (w t)+\alpha_{l} g(t)\right) \\
\varphi_{r}(t) & =\frac{\pi}{4}\left(\sin (w t)+\alpha_{r} g(t)\right)
\end{aligned}
$$

where $\left(\gamma, \alpha_{l}, \alpha_{r}\right) \in[-1,1]$ are the tunable kinematic parameters, and the subscript $r$ and $l$ stand for right and left wing, respectively.

As an example, Figure 5a shows both left and right wing motions resulted from choosing the parameters as $\gamma=1$, $\alpha_{l}=1$, and $\alpha_{r}=-1$. Also plotted is the additional term $g(t)$. We can see that with this parameterization, continuity is ensured at the end of wingbeats, while wing motions can be manipulated in the middle of each wingbeat.

The thorax input torques corresponding to the desired wing motions can be calculated in a similar manner as in 10. This approach is equivalent to feed-forward open loop 

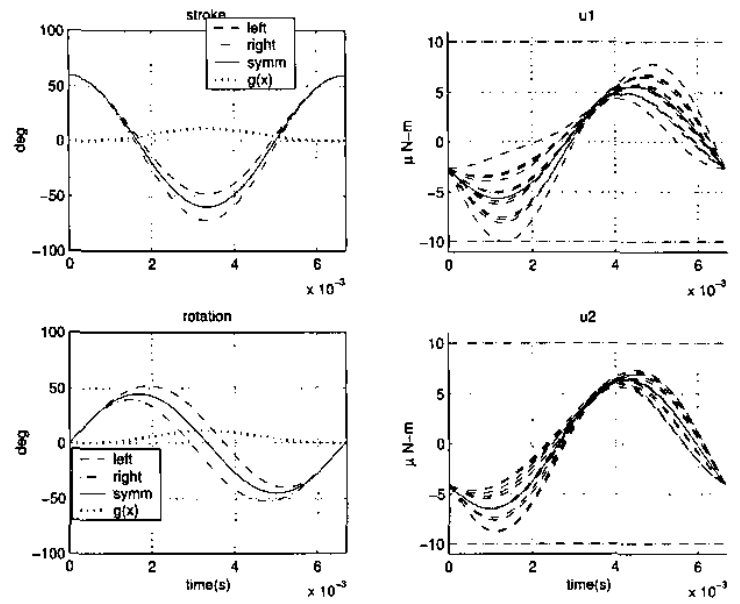

Fig. 5. (a) Left plots: Wing motions results from $\left(\gamma, \alpha_{l}, \alpha_{r}\right)=$ $(1,1,-1)$. The solid line traces correspond to the symmetric motion $\left(\gamma, \alpha_{l}, \alpha_{r}\right)=(0,0,0)$. (b) Right plots: Input torques for a family of permutations of the parameters $\left(\gamma, \alpha_{i}, \alpha_{r}\right) \in\{-1,1\}$. The horizontal lines correspond to the input saturation limits.

control of wing trajectories in a single wingbeat. Figure $5 \mathrm{~b}$ plots in detail a family of input torques obtained by varying the parameters $\left(\gamma, \alpha_{l}, \alpha_{r}\right)$ between $(-1,1)$. We can see that the inputs are always bounded by $\pm 10 \mu \mathrm{Nm}$.

To test whether the above parameterization scheme can generate desired aerodynamic torques to steer the insect body, we simulate through VIFS [10] to find the empirical map from wing kinematic parameters to the average body torques generated over a single wingbeat. Figure 6 and Figure 7 show the simulation results. It is seen that $\tau_{\text {pitch }}^{\hat{y}}$ and $\tau_{\text {yaw }}$ can be approximated with linear functions of $\alpha_{l}$ and $\alpha_{r}$, while $\tau_{\text {roll }}^{\wedge}$ with a linear function of $\gamma$ only. Therefore the mean torques are decoupled for the identification and control purposes. Figure 6 also shows the mean lift force as an approximate linear function of frequency.

\section{MODEL IDENTIFICATION}

The analysis in the previous section provide us with a torque decoupling scheme, together with a set of feasible control inputs i.e. wing kinematic parameters. Since we are interested in the insect dynamics close to the hovering regime where angular deviations and angular velocities are small, we linearize the dynamics (1) near hover and average it within a single wingbeat. For the purpose of designing a simple feedback controller, we approximate the actural continuous-time nonlinear system with a discretetime LTI model:

$$
\begin{array}{cl}
x(k+1) & =A x(k)+B u(k)+w(k) \\
y(k) & =x(k)+v(k)
\end{array}
$$
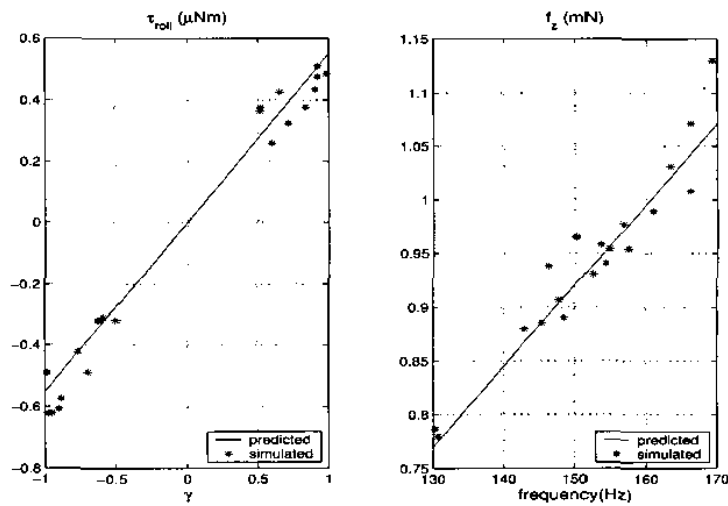

Fig. 6. Average roll torque, $\tau_{\text {roll }}$ (left) as a function of the parameter $\gamma$. Mean lift, $f_{z}$ (right) as a function of the wingbeat frequency. The simulated points are obtained by randomly select the values for the parameters $\left(\gamma, \alpha_{l}, \alpha_{r}\right)$.

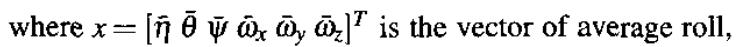
pitch, yaw angles and angular rates over one wingbeat;

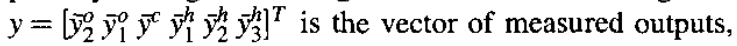
$w$ and $v$ represents model uncertainties, disturbances, and measurement noise, and $u=\left[u_{1} u_{2} u_{3}\right]^{T}=\left[\gamma \alpha_{l} \alpha_{r}\right]^{T}$ are the control inputs.

The matrices $[A, B]$ can be obtained directly from MFI morphological parameters such as mass, moment of inertia, center of mass, etc. However, these parameters are difficult to obtain in practice. Moreover, this approach cannot model the effect of the time varying part of the aerodynamic forces. An alternative approach is to run a large number of experiments and record the pair $[y(k), u(k)]$, and then find the matrices $[A, B]$ that best fit the data. In this work we recast the model identification problem into a least square solution to an overdetermined set of linear equations. The experiments were performed on the Virtual Insect Flight Simulator (VIFS), developed by the authors to provide a software testbed for insect flight [10]. The inputs and initial conditions are generated from random signals near trim condition, and the outputs are the sensor measurements.

Estimation of the system parameters and further investigation into the system dynamics in Equation (12) results in the following approximate parameter structures:

$$
A=\left[\begin{array}{rr}
I_{3 \times 3} & T I_{3 \times 3} \\
A_{21} & A_{22}
\end{array}\right] \quad B=\left[\begin{array}{r}
0_{3 \times 3} \\
B_{22}
\end{array}\right]
$$

where $T$ is the wingbeat period. As expected, it was found that $A_{22}$ matrix is close to an identity matrix. The structure of the $B_{22}$ matrix also reflects our previous torque decoupling scheme through wing kinematic parameterization. For model validation, Figure 8 plots mean angle and angular rates predicted by the LTI model together the simulation results from VIFS for a consecutive 50 

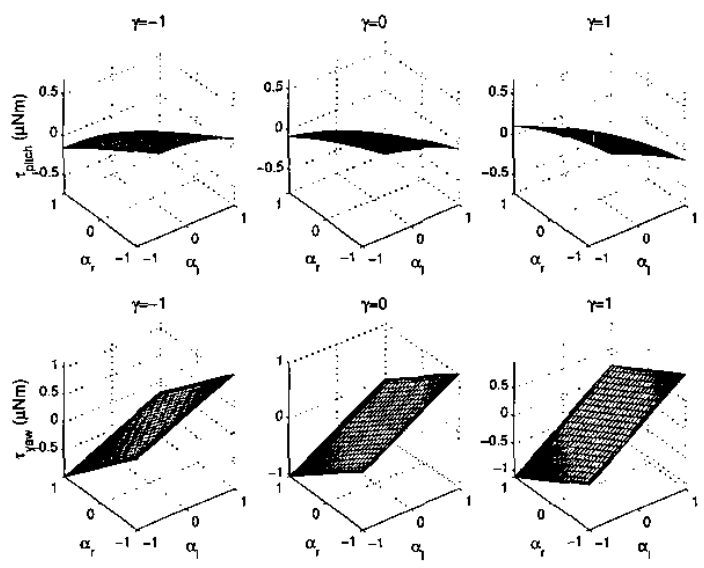

Fig. 7. Average pitch and yaw torques as a linear map of the parameters $\alpha_{l}$ and $\alpha_{r}$

wingbeats. It can be seen that the predicted values match the simulated ones very well.

\section{LQR CONTROLLER DESIGN}

Based on the identified model found above, stabilizing output feedback control laws are designed and tuned first on the nominal LTI model, then tested on the fully nonlinear continuous time system of Equations (1).

In this work, a output feedback $L Q R$ regulator $u=-K y$ was designed to minimizes the following quadratic cost function

$$
J=\lim _{N \rightarrow \infty} E\left(\sum_{k=1}^{N} x(k)^{T} Q x(k)+u(k)^{T} R u(K)\right)
$$

where $Q \geq 0$ and $R>0$ are the weighting matrices to reflect the trade-off between regulation performance and control effort, and the diagonal entries in the weighting matrices are iteratively tuned to ensure a good transient response without saturating the control inputs. The final choice of the the weighting matrices $Q$ and $R$ for the regulator are $Q=\operatorname{diag}(10,20,20,1,1,1)$ and $R=\operatorname{diag}(1,2,5)$.

The LQR controller was finally tested on the fully nonlinear continuous time model which includes the MFI dynamic model (1), the thorax dynamic model (9), and the sensors models described in Section III. The simulations are based on an MFI of $100 \mathrm{mg}$ and $2 \mathrm{~cm}$ tip-to-tip wingspan with wingbeat frequancy $f=150 \mathrm{~Hz}$.

Figure 9 shows the simulation of the MFI steering $90^{\circ}$ degree in yaw angle. The $\mathrm{LQR}$ controller drives the MFI smoothly to the desired position in approximately 50 wingbeats, i.e. approximately one third of a second. Note that the pitch velocity (center middle plot) exhibits a highly oscillatory behavior. This is due to the unavoidable periodic pitch torque from the flapping of the wings. Also depicted in the figures are the sensor outputs of the MFI

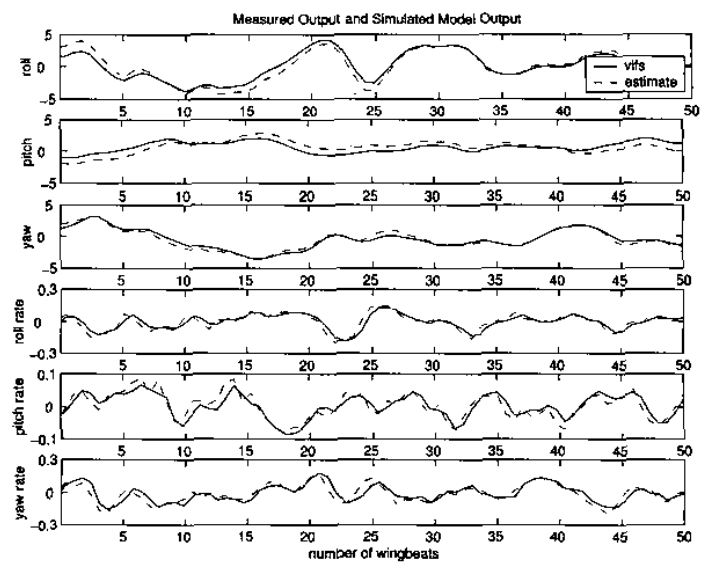

Fig. 8. Comparison of the predicted mean angles and angular velocities from the nominal LTI model(dashed line) and those simulated from VIFS (solid line) over 50 consecutive wingbeats; $\gamma, \alpha_{l}$, and $\alpha_{r}$ are chosen randomly.

angles and angular velocities. The halteres estimates the mean angular velocities remarkably well by filtering out the high amplitude time varying disturbance due to the wing flapping. The ocelli track the roll and pitch angles correctly. The MEMS compass initially underestimates the yaw angle, but provides the correct error sign, and eventually tracks the yaw correctly when the the angle becomes small. The bottom plots of Figure 9 present the corresponding control inputs to the thorax actuators. As expected, the control inputs never exceed the torque limits and they are continuous at the end of every wingbeat.

Figure 10 shows the MFI recovering from an upside down $180^{\circ}$ roll angle. Again, the LQR controller drives the MFI smoothly to the desired position in approximately 75 wingbeats, which correspond to half a second. In this situation, when the roll and pitch angles are very large, the ocelli fail to estimate them exactly. However it was shown in [4] that they can always provide a signal that recovers the MFI to hovering. The bottom plots in Figure 10 present the corresponding wing kinematic parameters chosen as inputs. They are strongly related to the control effort required by the LQR controller. The parameter $\gamma$, which is directly related to roll body torque, saturates, thus implying an aggressive maneuvering, and infact the MFI roll angle exhibit some overshooting as it in general common with controller with high gains. On the other hand, the LQR gains were designed for small angle maneuvers, and anti wind-up techniques for large angles maneuvers will be explored in the future. 

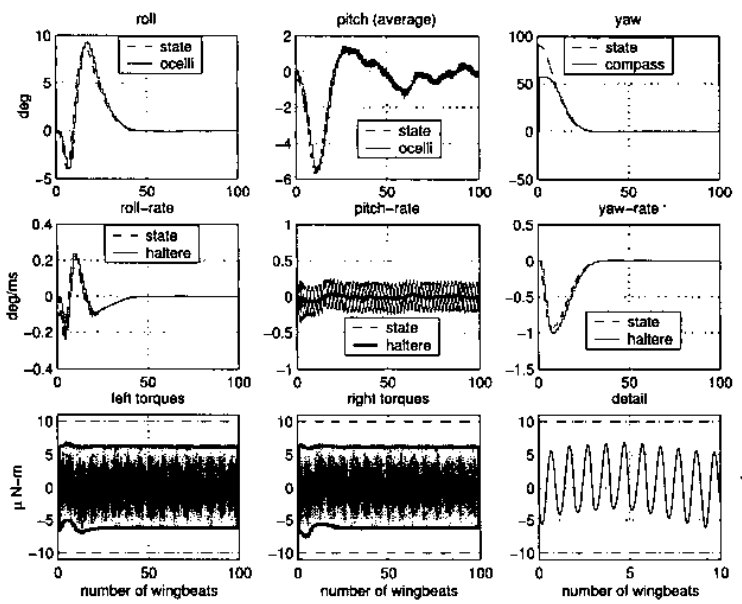

Fig. 9. Simulation of MFI evolution and sensor outputs during $90^{\circ}$ steering. (Top plots): Roll, $\phi$, pitch, $\theta$, and yaw, $\psi$ angles. (Middle plots): $\omega_{x}$, pitch, $\omega_{y}$, and yaw, $\omega_{z}$, angular velocities. (Bottom plots): Actuator control inputs $\left(u_{1}, u_{2}\right)$ for left (left plot) and right (center plot) wing. The bottom right plot shows a detail of the torque $u_{1}$ of the left wing for the first 10 wingbeats. Time is expressed in wingbeats, i.e. 1 wingbeat $=6.7 \mathrm{~ms}$

\section{CONCLUSION}

In this work, high level attitude control of the MFI was considered. Based on recently developed thorax model, wing kinematic parameterization method was developed. A nominal state-space LTI model in hover was identified through linear estimation and a LQR controller was designed. Sensor models such as haltere, magnetic compass, and ocelli were included inside the closed loop system and the simulations show a performance comparable to that of real insect that can complete a full maneuver in approximately $30-50$ wingbeats. It is also shown that under LQR control the MFI is able to recover from large angular displacements such as recovering from an upside down orientation and steering $90^{\circ}$ degrees in the yaw axis with fast transient response, despite the fact the LQR controller was designed for small angular errors and the sensor outputs are nonlinear for large angles.

Future work involves quantification of the parameter uncertainties in our nominal model, due to sensors noise, atmospheric turbulence, and most of all undermodeling of nonlinear periodic signals. Given the limited computational resources of the MFI, we will address the problem of reducing complexity of the controller, for example by controlling the wing motions every $\mathrm{N}$-wingbeats. Extension to the complete 6 DOF system dynamics including position control is under investigation in hovering and forward flight.
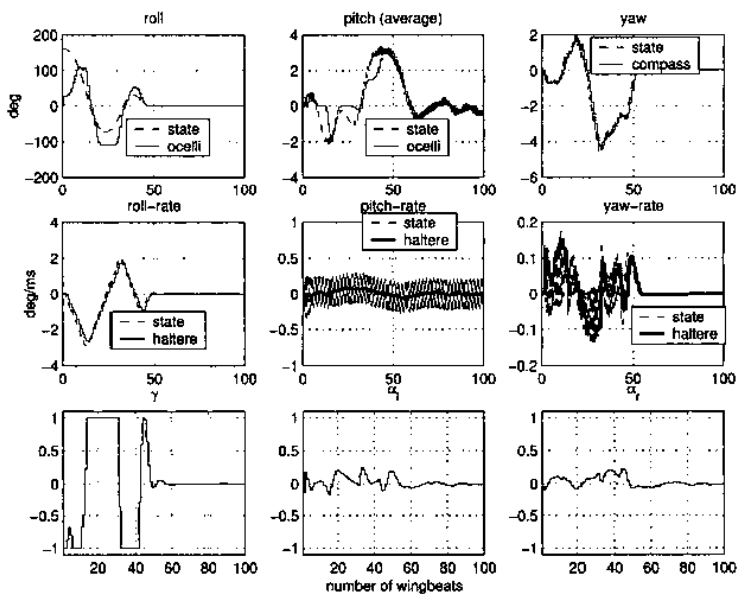

Fig. 10. Simulation of MFI evolution and sensor outputs during recovering from upsidedown orientation. (Bottom plots): Wing kinematic parameters $\left(\gamma, \alpha_{r}, \alpha_{l}\right)$.

\section{REFERENCES}

[1] B. Motazed, D. Vos, and M. Drela, "Aerodymatics and flight control design for hovering MAVs," in Proc of Amer Control Conference, Philadelphia, PA, June 1998.

[2] R.S. Fearing, K.H. Chiang, M.H. Dickinson, D.L. Pick, M. Sitti, and J. Yan, "Transmission mechanism for a micromechanical flying insect," in Proc. of ICRA, 2000.

[3] X. Deng, L. Schenato, and S.S. Sastry, "Model identification and attitude control scheme for a micromechanical flying insect," in Proc. of the 7th ICARCV, 2002.

[4] L.Schenato, W.C. Wu, and S.S. Sastry, "Attitude control for a micromechanical flying insect via sensor output feedback," Submitted to IEEE Journal of Robotics and Automation.

[5] W.C. Wu, L. Schenato, R.J. Wood, and R.F. Fearing, "Biomimetic sensor suite for flight control of a micromechanical flight insect: Design and experimental results," in Submitted to ICRA, Taipei, Taiwan, 2003.

[6] H. Schuppe and R. Hengstenberg, "Optical properties of the ocelli of calliphora erythrocephala and their role in the dorsal light response," Joumal of Comparative Biology A, vol. 173, pp. 143-149, 1993.

[7] R. Hengstenberg, "Mechanosensory control of compensatory head roll during flight in the blowfly Calliphora erythrocephala Meig," Journal of Comparative Physiology $A$, vol. 163, pp. 151-165, 1988.

[8] G. Nalbach, "The halteres of the blowfly Calliphora: I. kinematics and dynamics," Journal of Comparative Physiology A, vol. 173, pp. 293-300, 1993.

[9] S. Avadhanula, R. J. Wood, D. Campolo, and R. S. Fearing, "Dynamically tuned design of the mfi thorax," in Proc. of ICRA, 2002.

[10] L. Schenato, X. Deng, W.C. Wu, and S.S. Sastry, "Virtual insect flight simulator (vifs): A software testbed for insect flight," in Proc. of ICRA, 2001. 